Physica 96C (1979) 391-409

(C) North-Holland Publishing Company

\title{
TIME AND FREQUENCY DEPENDENCE OF NEARLY RESONANT LIGHT SCATTERED FROM COLLISIONALLY PERTURBED ATOMS
}

\author{
G. NIENHUIS \\ Fysisch Laboratorium, Ruksuniversiteit Utrecht, Postbus 80 000, 3508 TA Utrecht, The Netherlands
}

Recelved 2 November 1978

\begin{abstract}
We generalize previous theories of collisional redistribution of near-resonance radiation to the case of a tume-dependent incident intensity. In the absence of saturation the relation between the incident and the scattered spectrum is given by a tıme-dependent redistribution function In the special case of an incident pulse with an exponential rise and decay we evaluate the time-dependent strength of the Rayleigh or Raman scattering and of the fluorescence We pay special attention to the restriction on the time resolution resulting from the spectral resolution that is necessary to observe separately the two peaks in the scattered spectrum.
\end{abstract}

\section{Introduction}

With the advent of intense tunable lasers it has become feasible to observe the spectral redistribution of light scattered from an atomic vapor after arradiation in the far wing of a spectral line. Experiments of this type have been reported by Carlsten and coworkers [1-3]. A theoretical description of redistribution by collisions has been given by Huber [4] and by Omont et al. [5]. These treatments employ the impact limit, which is applicable in the central part of the absorption line. We have given a generalized treatment, allowing for irradiation outside the impact region [6-9]. In refs. 4-9 the scattered-light intensity is evaluated as a function of the incident and the scattered frequency, in the case of a stationary incident intensity. For far-wing irradiation the peaks corresponding to Rayleıgh or Raman scatterıng and to collision-induced fluorescence become well separated in the scattered spectrum.

The complementary problem of the time dependence of the scattered light has also received attention recently. Rousseau and coworkers [10-12] observed the time dependence of the fluorescence of iodine molecules after far-wing irradiation with a short laser pulse. These authors observed a rapidly decaying component, which reflects the decay of the pulse, and a more slowly decaying component corresponding to the lifetime of the excited state. The rapid decay was attributed to Raman scattering, the slow decay to collision-induced fluorescence. This is in line with the observation that the slowly decaying component is enhanced by increasing the pressure. A theoretical description of these effects has been given in the impact limit by Mukamel et al. [13]

In a most interesting recent paper Courtens and Szoke [14] have given a clarifying description of the scattered spectrum, accounting both for spectral and tıme resolution. In the impact limit they obtained expressions for the strengths (not the profiles) of the various peaks in the spectrum, allowing for high-intensity effects such as saturation and the dynamic Stark effect. These expressions are valid in the adiabatic case that the rate of change of the incident intensity is smaller than the frequency separation of the peaks in the scattered spectrum.

In the present paper we describe the intensity of the light scattered from atoms perturbed by collisions as a function of time and of the scattered frequency. We avold the restrictions of the impact limit, which is necessary to include the experimentally important case of irradiation in the far wing of an absorption line However, we do not account for saturation effects. The method is a generalization of our previous work [6-9]. We obtain expressions for the time-dependent strengths of the Rayleigh or Raman scattering and of the fluorescence in terms of 
the characteristics of the pulse. The restrictions on the time resolution resulting from the use of a narrow-band frequency filter are properly accounted for.

\section{The significance of time-dependent spectra}

Determination of the frequency spectrum of a time-dependent signal with a resolution $\Delta \omega$ requires the observation of the signal during a time $\Delta \omega^{-1}$. Thus if the spectrum of a non-stationary signal is recorded by measuring the transmission through a tunable filter with bandwidth $\Delta \omega$, the time resolution cannot be better than $\Delta \omega^{-1}$. The concept of a time-dependent spectrum is not a property of the signal alone, rather it refers to the combination of the signal and the frequency filter.

A careful study of the significance of time-dependent spectra has recently been given by Eberly and Wódkiewicz [15]. In the spirit of their work we derive a simple expression for the time-dependent spectrum that is suitable for the purpose of this paper. We consider a non-stationary electromagnetic field. Its electric-field component $E(t)$ may be decomposed in its positive- and negative-frequency parts $E^{(+)}(t)$ and $E^{(-)}(t)$, according to the definitions

$$
E(t)=E^{(+)}(t)+E^{(-)}(t)
$$

where

$$
E^{(+)}(t)=\frac{1}{2 \pi} \int_{0}^{\infty} \mathrm{d} \omega \mathrm{e}^{-1 \omega t} \tilde{E}(\omega)
$$

and

$$
E^{(-)}(t)=\frac{1}{2 \pi} \int_{-\infty}^{0} \mathrm{~d} \omega \mathrm{e}^{-1 \omega t} \tilde{E}(\omega)=E^{(+)}(t)^{*}
$$

Here

$$
\tilde{E}(\omega)=\int_{-\infty}^{\infty} \mathrm{d} t \mathrm{e}^{1 \omega t} E(t)
$$

1s the Fourner transform of the electric field. By the definitions (2.2) and (2.3) the Fourner transform of $E^{(+)}(t)$ is equal to $\tilde{E}(\omega) \theta(\omega)$, and $E^{(-)}(t)$ has $\tilde{E}(\omega) \theta(-\omega)$ as its Fourier transform, where $\theta$ is the unit step function. The spectral distribution of the incident electromagnetic energy per unit area until the time $t$ is $[15,16]$

$$
G(\omega, t)=\frac{c}{4 \pi^{2}} \int_{-\infty}^{t} \mathrm{~d} t_{1} \int_{-\infty}^{t} \mathrm{~d} t_{2} \mathrm{e}^{-1 \omega\left(t_{1}-t_{2}\right)}\left\langle E^{(-)}\left(t_{1}\right) \cdot E^{(+)}\left(t_{2}\right)\right\rangle .
$$

The brackets \langle\rangle in (2.5) denote an ensemble average over the statistical distribution of the electromagnetic field, both for a classical treatment, where $E(t)$ is a stochastic function, and for a quantum-mechanical treatment where $E(t)$ is a Heisenberg operator, with $E^{(+)}$and $E^{(-)}$as its absorptive and emissive parts. From the double time integral in (2.5) it is apparent that the spectral energy of a signal during a tıme interval from $t_{1}$ to $t_{3}$ is not just the sum of the spectral energy of the signal as observed from $t_{1}$ to $t_{2}$, and from $t_{2}$ to $t_{3}$. This illustrates that photons emitted in different time intervals can interfere. Accordingly, it is not justified in general to identify the time denvative of 
$G$ with the time-dependent spectrum, as has been noted in ref. 15. In fact, from the explicit expression of this tıme derivative

$$
g(\omega, t)=\frac{\partial G(\omega, t)}{\partial t}=\frac{c}{2 \pi^{2}} \operatorname{Re} \int_{0}^{\infty} \mathrm{d} \tau \mathrm{e}^{-1 \omega \tau}\left\langle E^{(-)}(t) \cdot E^{(+)}(t-\tau)\right\rangle,
$$

one readily checks that $g$ may well be partly negative in special cases On the other hand, the integral of (2.6) over frequency is equal to the incident radiative power per unit area

$$
\int_{-\infty}^{\infty} \mathrm{d} \omega g(\omega, t)=\frac{c}{2 \pi}\left\langle E^{(-)}(t) \cdot E^{(+)}(t)\right\rangle
$$

The integral of $g$ over time gives the spectral distribution of the total energy per unit area

$$
\int_{-\infty}^{\infty} \mathrm{d} t g(\omega, t)=G(\omega, \infty)=\frac{c}{4 \pi^{2}}\left\langle|\tilde{E}(\omega)|^{2}\right\rangle
$$

Furthermore it is obvious from (2.6) that for a stationary signal, $g$ becomes equal to the correct stationary spectrum as given by the Wiener-Khintchine relation $[15,17]$.

We intend to derive a relation between the physical time-dependent spectrum as measured by using a highresolution spectrometer, and the function $g(\omega, t)$, which is defined by $(2.6)$ in terms of the signal alone. First we give an additional relation between the function $g$ and the correlation function of the electric field. From (2.5) one derives directly

$$
\int_{-\infty}^{\infty} \mathrm{d} \omega \mathrm{e}^{1 \omega \tau} G(\omega, t)=\frac{c}{2 \pi} \int_{-\infty}^{t} \mathrm{~d} t_{1}\left\langle E^{(-)}\left(t_{1}\right) \cdot E^{(+)}\left(t_{1}-\tau\right)\right\rangle \text { for } \tau>0 .
$$

Differentiation of (2.9) with respect to $t$ gives the identity

$$
\int_{-\infty}^{\infty} \mathrm{d} \omega \mathrm{e}^{1 \omega \tau} g(\omega, t)=\frac{c}{2 \pi}\left\langle E^{(-)}(t) \cdot E^{(+)}(t-\tau)\right\rangle \quad \text { for } \tau>0 .
$$

When using a spectrometer, the physically observed signal is found by multiplying each Fourier component $\tilde{E}(\omega)$ with the transfer function $\tilde{s}(\omega)$ characterizing the spectrometer $[14,15]$. Hence the Fourier transform of the filtered field is

$$
\tilde{E}_{\mathrm{D}}(\omega)=\tilde{s}(\omega) \tilde{E}(\omega)
$$

The central frequency $\omega_{D}$ of $\tilde{s}(\omega)$ is the setting frequency, and the width $\Gamma$ of $\tilde{s}$ is the passband width of the filter In practical cases $\Gamma$ is much smaller than $\omega_{D}$, which is tuned over the frequency range of the signal. For these large positive frequencies we may write

$$
\tilde{s}(\omega)=\tilde{v}\left(\omega-\omega_{\mathrm{D}}\right), \quad \omega \gg \Gamma,
$$

where the shifted transfer function $\tilde{v}$ is centered around the origin. Because of causality the observed filtered field 
at tıme $t$ can only depend upon the signal field at earlier tımes. Therefore the tıme-dependent filter function

$$
v(\tau)=\frac{1}{2 \pi} \int_{-\infty}^{\infty} \mathrm{d} \omega \tilde{v}(\omega) \mathrm{e}^{-1 \omega \tau}
$$

must be zero for negative values of $\tau$ After takıng the Fourier transform of the positive-frequency part of (2.11), and applying the convolution theorem, we obtain

$$
E_{\mathrm{D}}^{(+)}(t)=\int_{0}^{\infty} \mathrm{d} \tau \mathrm{e}^{-1 \omega_{\mathrm{D}} \tau} v(\tau) E^{(+)}(t-\tau)
$$

The negative-frequency part $E_{\mathrm{D}}^{(-)}(t)$ is the complex conjugate of (2.14).

The physically measured time-dependent spectrum is the time-dependent energy of the filtered field, which according to (2.14) depends upon the setting frequency $\omega_{D}[15]$. Hence we write

$$
g_{\mathrm{D}}\left(\omega_{\mathrm{D}}, t\right)=\frac{c}{2 \pi}\left\langle E_{\mathrm{D}}^{(-)}(t) \cdot E_{\mathrm{D}}^{(+)}(t)\right\rangle
$$

It is this quantity that determines the counting rate of a photodetector behind the spectrometer. Several explicit expressions of $g_{\mathrm{D}}$ in terms of the signal-field correlation function have been given by Eberly and Wódkiewicz [15]. We wish to express $g_{D}$ directly in terms of $g$. After substituting (2.14) and its complex conjugate in (2.15), we use (2.10) to express the correlation function in terms of $g$. The result after a simple change of integration variables is

$$
g_{\mathrm{D}}\left(\omega_{\mathrm{D}}, t\right)=2 \operatorname{Re} \int_{0}^{\infty} \mathrm{d} \tau \int_{0}^{\infty} \mathrm{d} \tau^{\prime} \int_{-\infty}^{\infty} \mathrm{d} \omega \mathrm{e}^{-1 \omega \tau^{\prime}} v^{*}(\tau) v\left(\tau+\tau^{\prime}\right) g\left(\omega_{\mathrm{D}}-\omega, t-\tau\right)
$$

For convenience we normalize $v$ in such a way that $g_{\mathrm{D}}$ and $g$ have the same total strength when integrated over time and frequency. This requires the normalization condition

$$
\int_{0}^{\infty} \mathrm{d} \tau|v(\tau)|^{2}=\frac{1}{2 \pi}
$$

(One notices that eq. (2.17) causes $E_{\mathrm{D}}$ to have a dimension different from that of $E$, but $g_{\mathrm{D}}$ now has the proper dimension of a spectral power per unit area.)

For realistic spectrometers (e.g. a Fabry-Perot interferometer) the filter function $v(\tau)$ is given by a simple exponential [15]

$$
v(\tau)=\left(\frac{\Gamma}{2 \pi}\right)^{\frac{1}{2}} \mathrm{e}^{-\Gamma \tau / 2} \theta(\tau)
$$

In this case eq. (2.16) attains the form

$$
g_{\mathrm{D}}\left(\omega_{\mathrm{D}}, t\right)=\int_{0}^{\infty} \mathrm{d} \tau \Gamma \mathrm{e}^{-\Gamma \tau} \int_{-\infty}^{\infty} \mathrm{d} \omega \frac{\Gamma / 2 \pi}{(\Gamma / 2)^{2}+\omega^{2}} g\left(\omega_{\mathrm{D}}-\omega, t-\tau\right) .
$$

This expression relates the physically observed tıme-dependent spectrum $g_{\mathrm{D}}$ to the quasi-spectrum $g$, defined by 
(2.6). It is apparent from (2.19) that the passband width $\Gamma$ of the filter affects the spectrum in a twofold way. Firstly, the function $g$ is averaged over the recent past as indicated by the normalized time-distribution function $\Gamma \mathrm{e}^{-\Gamma \tau}$. This excludes the time resolution from being better than $\Gamma^{-1}$. Secondly, the function $g$ is averaged over a Lorentzian frequency window with a width $\Gamma$, which determines the frequency resolution. We feel that the interdependence of time and spectral resolution is illustrated in (2.19) with greater lucidity than in expressions given in ref. 15.

\section{Time-dependent fluorescence spectrum}

We consider the situation of a pulse of radiation incident on a vapor of atoms in a perturber gas. The electricfield component of the radiation at the position $R$ of a scattering atom has a positive-frequency part $E^{(+)}(R, t)$ The $R$-dependence of $E^{(+)}$may be factored out by writing

$$
E^{(+)}(R, t)=\mathrm{e}^{1 k_{1} \cdot R} \epsilon_{1} E^{(+)}(t)
$$

where $k_{1}$ and $\epsilon_{1}$ are the wave vector and the unit polarization vector of the incident radiation, and $E^{(+)}(t)$ is the positive frequency part of the magnitude of the electric field at the origin. The atom is excited by absorption from an initial state $|1\rangle$ to an excited state $|e\rangle$, and decays to a final state $|f\rangle$ by subsequent emission of radiation. As before we introduce the simplified notation [6]

$$
\begin{aligned}
& d_{1}=\mu_{\mathrm{el}} \cdot \epsilon_{1} \mathrm{e}^{1 k_{1} \cdot R}, \\
& d_{2}=\mu_{\mathrm{ef}} \cdot \epsilon_{2} \mathrm{e}^{1 k_{2} \cdot R},
\end{aligned}
$$

where $\boldsymbol{u}_{\mathrm{e} 1}$ and $\boldsymbol{u}_{\mathrm{ef}}$ denote the atomic electric-dipole operator between the indicated states. The states $|\mathrm{i}\rangle,|\mathrm{e}\rangle$ and $|\mathrm{f}\rangle$ may consist of several degenerate or nearly degenerate substates From a direct generalization of the derivation in ref. 6 we obtain an expression for the spectral distribution $G_{2}\left(\omega_{2}, t\right)$ of the energy of the radiation with wave vector $k_{2}$, with frequency $\omega_{2}$ and with polarization $\epsilon_{2}$, scattered before the time $t$ within a unit solid angle by a unit volume of the system with a density $n_{\mathrm{A}}$ of scattering atoms. The result is

$$
\begin{aligned}
G_{2}\left(\omega_{2}, t\right)= & n_{\mathrm{A}} \frac{\omega_{2}^{4}}{4 \pi^{2} \hbar^{2} c^{3}} 2 \operatorname{Re} \int_{-\infty}^{t} \mathrm{~d} t_{4} \int_{-\infty}^{t_{4}} \mathrm{~d} t_{3} \int_{-\infty}^{t_{4}} \mathrm{~d} t_{2} \int_{-\infty}^{t_{2}} \mathrm{~d} t_{1} \mathrm{e}^{-1 \omega_{2}\left(t_{4}-t_{2}\right)}\left\langle E^{(-)}\left(t_{3}\right) E^{(+)}\left(t_{1}\right)\right\rangle \\
& \times \operatorname{Tr} \mathrm{e}^{-1 H\left(t-t_{2}\right) / \hbar} d \mathrm{e}^{-1 H\left(t_{2}-t_{1}\right) / \hbar} d_{1} \mathrm{e}^{-1 H t_{1} / \hbar} \rho \mathrm{e}^{\mathrm{i} H t_{3} / \hbar} d_{1}^{\dagger} \mathrm{e}^{1 H\left(t_{4}-t_{3}\right) / \hbar} d_{2} \mathrm{e}^{1 H\left(t-t_{4}\right) / \hbar},
\end{aligned}
$$

where $H$ is the hamiltonian of the particle system of one scattering atom in a perturber bath, and $\rho$ is the initial equilibrium density matrix of the particle system. Eq. (3.4) is valid to first order in the irradiated intensity. The four integration time variables $t_{1}, t_{2}, t_{3}$ and $t_{4}$ in (3.4) can attain values corresponding to three possible time ordenngs (1) $t_{1}<t_{2}<t_{3}<t_{4}$, (11) $t_{1}<t_{3}<t_{2}<t_{4}$, (11) $t_{3}<t_{1}<t_{2}<t_{4}$. As in ref. 6 we call $\tau_{1}, \tau_{2}$ and $\tau_{3}$ the successive time intervals separated by these variables. This gives rise to an expression for $G_{2}$ as a sum of three terms

$$
\begin{aligned}
G_{2}\left(\omega_{2}, t\right)= & n_{\mathrm{A}} \frac{\omega_{2}^{4}}{4 \pi^{2} \hbar^{2} c^{3}} 2 \operatorname{Re} \int_{-\infty}^{t} \mathrm{~d} t_{4} \int_{0}^{\infty} \mathrm{d} \tau_{1} \mathrm{~d} \tau_{2} \mathrm{~d} \tau_{3} \\
& \times\left[\mathrm{e}^{-\mathrm{t} \omega_{2}\left(\tau_{2}+\tau_{3}\right)}\left\langle E^{(-)}\left(t_{4}-\tau_{3}\right) E^{(+)}\left(t_{4}-\tau_{1}-\tau_{2}-\tau_{3}\right)\right\rangle \operatorname{Tr} d_{2} \mathrm{e}^{-1 L \tau_{3}}\left\{\left[\mathrm{e}^{-1 L \tau_{2}}\left(d_{2}^{\dagger} \mathrm{e}^{-\mathrm{i} L \tau_{1}}\left(d_{1} \rho\right)\right)\right] d_{1}^{\dagger}\right\}\right.
\end{aligned}
$$




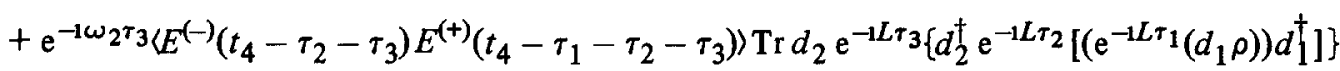

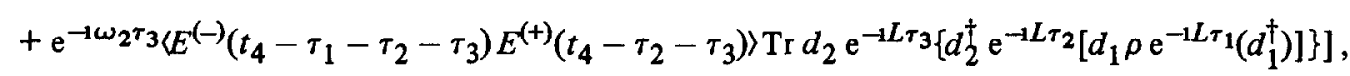

where the Liouville operator is defined to include the effect of the radiative decay rate $\Gamma_{\mathrm{a}}$ of the atomic state $|\mathrm{a}\rangle$, according to the relation

$$
-1 L \rho_{\mathrm{ab}}=-\frac{1}{\hbar}\left[H, \rho_{\mathrm{ab}}\right]-\frac{1}{2}\left(\Gamma_{\mathrm{a}}+\Gamma_{\mathrm{b}}\right) \rho_{\mathrm{ab}}
$$

It is attractive to separate in the expression for $G_{2}$ the effect of the incident radiation from the effects of the particle system We introduce $G_{1}\left(\omega_{1}, t\right)$ as the spectral energy distribution per unit area of the incident radiation until the time $t$. This quantity is related to the correlation function $\left\langle E^{(-)}\left(t_{1}\right) E^{(+)}\left(t_{1}-\tau\right)\right\rangle$ of the incident field by expressions similar to (2.5) and (29) After shifting the integration variable $t_{4}$ and substituting (29) 1nto (3.5) we obtain

$$
G_{2}\left(\omega_{2}, t\right)=n_{\mathrm{A}} \frac{\omega_{2}^{4}}{2 \pi \hbar^{2} c^{4}} \int_{-\infty}^{\infty} \mathrm{d} \omega_{1} \int_{0}^{\infty} \mathrm{d} \tau f\left(\omega_{2}, \omega_{1} \mid \tau\right) G_{1}\left(\omega_{1}, t-\tau\right)
$$

where the time-dependent redistribution function $f$ is equal to

$$
\begin{aligned}
f\left(\omega_{2}, \omega_{1} \mid \tau\right)= & 2 \operatorname{Re} \int_{0}^{\infty} \mathrm{d} \tau_{1} \int_{0}^{\infty} \mathrm{d} \tau_{2} \mathrm{e}^{1 \omega_{1} \tau_{1}+1\left(\omega_{1}-\omega_{2}\right) \tau_{2}-1 \omega_{2} \tau} \\
& \times \operatorname{Tr} d_{2} \mathrm{e}^{-1 L \tau}\left\{\left[\mathrm{e}^{-1 L \tau_{2}}\left(d_{2}^{\dagger} \mathrm{e}^{-\mathrm{i} L \tau_{1}}\left(d_{1} \rho\right)\right)\right] d_{1}^{\dagger}\right\} \\
& +2 \operatorname{Re} \int_{0}^{\infty} d \tau_{1} \int_{0}^{\tau} \mathrm{d} \tau_{3}\left[\mathrm{e}^{\mathrm{i} \omega_{1} \tau_{1}-1 \omega_{2} \tau_{3}} \operatorname{Tr} d_{2} \mathrm{e}^{-1 L \tau_{3}}\left\{d_{2}^{\dagger} \mathrm{e}^{-1 L\left(\tau-\tau_{3}\right)}\left[\left(\mathrm{e}^{-1 L \tau_{1}}\left(d_{1} \rho\right)\right) d_{1}^{\ddagger}\right]\right\}\right. \\
& \left.+\mathrm{e}^{-1 \omega_{1} \tau_{1}-1 \omega_{2} \tau_{3}} \operatorname{Tr} d_{2} \mathrm{e}^{-1 L \tau_{3}}\left\{d_{2}^{\dagger} \mathrm{e}^{-1 L\left(\tau-\tau_{3}\right)}\left[d_{1} \rho \mathrm{e}^{-1 L \tau_{1}}\left(d_{1}^{\dagger}\right)\right]\right\}\right] .
\end{aligned}
$$

If we introduce the quasi-spectra $g_{1}$ and $g_{2}$ according to

$$
g_{1}\left(\omega_{1}, t\right)=\frac{\partial G_{1}\left(\omega_{1}, t\right)}{\partial t}, \quad g_{2}\left(\omega_{2}, t\right)=\frac{\partial G_{2}\left(\omega_{2}, t\right)}{\partial t}
$$

then we find immediately from (3.7)

$$
g_{2}\left(\omega_{2}, t\right)=n_{\mathrm{A}} \frac{\omega_{2}^{4}}{2 \pi \hbar^{2} c^{4}} \int_{-\infty}^{\infty} d \omega_{1} \int_{0}^{\infty} d \tau f\left(\omega_{2}, \omega_{1} \mid \tau\right) g_{1}\left(\omega_{1}, t-\tau\right)
$$

Eqs. (2.6) and (2.10) relate $g_{1}\left(\omega_{1}, t\right)$ to the correlation function of the electric-field component of the incident radiation. The physically observed tıme-dependent spectrum $g_{\mathrm{D}}\left(\omega_{2}, t\right)$ of the scattered radiation is found by substituting $g_{2}$ for $g$ in eq. (2.19). 
It is tempting to interpret $f\left(\omega_{2}, \omega_{1} \mid \tau\right)$ as measuring the probability for scattering a photon with frequency $\omega_{1}$ into a photon with frequency $\omega_{2}$, with a delay time $\tau$ between absorption and emission. This would lead one to introduce

$$
\frac{\partial^{3} \sigma\left(\omega_{2}, \omega_{1} \mid \tau\right)}{\partial \omega_{2} \partial \Omega \partial \tau}=\frac{\omega_{1} \omega_{2}^{3}}{2 \pi \hbar^{2} c^{4}} f\left(\omega_{2}, \omega_{1} \mid \tau\right)
$$

as the triple differentıal cross section for inelastic delayed photon scattering. This interpretation is justified only apart from the interdependence between time and frequency resolution as expressed by (2.19).

Eqs. (3.7), (3.10) and (3.8) express the main result of this section. The time-dependent redistribution function $f$ depends on the polarization and the direction of the incident and the scattered radiation. Simplified expressions arise if we integrate eq. (3.8) over time or over one of the two frequencles. We find

$$
\begin{aligned}
\int_{0}^{\infty} \mathrm{d} \tau f\left(\omega_{2}, \omega_{1} \mid \tau\right)= & 2 \operatorname{Re} \int_{0}^{\infty} \mathrm{d} \tau_{1} \int_{0}^{\infty} \mathrm{d} \tau_{2} \int_{0}^{\infty} \mathrm{d} \tau_{3} \\
& \times\left[\mathrm{e}^{1 \omega_{1} \tau_{1}+1\left(\omega_{1}-\omega_{2}\right) \tau_{2}-1 \omega_{2} \tau_{3}} \operatorname{Tr} d_{2} \mathrm{e}^{-1 L \tau_{3}}\left\{\left[\mathrm{e}^{-1 L \tau_{2}}\left(d_{2}^{\dagger} \mathrm{e}^{-1 L \tau_{1}}\left(\mathrm{~d}_{1} \rho\right)\right)\right] d_{1}^{\dagger}\right\}\right. \\
& +\mathrm{e}^{1 \omega_{1} \tau_{1}-1 \omega_{2} \tau_{3}} \operatorname{Tr} d_{2} \mathrm{e}^{-1 L \tau_{3}}\left\{d _ { 2 } ^ { \dagger } \mathrm { e } ^ { - 1 L \tau _ { 2 } } \left[\left(\mathrm{e}^{\left.\left.\left.-1 L \tau_{1}\left(d_{1} \rho\right)\right) d_{1}^{\dagger}\right]\right\}}\right.\right.\right. \\
& \left.+\mathrm{e}^{-1 \omega_{1} \tau_{1}-1 \omega_{2} \tau_{3}} \operatorname{Tr} d_{2} \mathrm{e}^{-1 L \tau_{3}}\left\{d_{2}^{\dagger} \mathrm{e}^{-1 L \tau_{2}}\left[d_{1} \rho \mathrm{e}^{-1 L \tau_{1}}\left(d_{1}^{\dagger}\right)\right]\right\}\right] \\
= & F\left(\omega_{2}, \omega_{1}\right),
\end{aligned}
$$

which is equal to the stationary redistribution as derived in ref. 6 . Hence, if the time-integrated fluorescence spectrum is recorded, the redistribution of the time-integrated incident spectrum is given by the redistribution function pertaining to the stationary case.

Integration of eq. (3.8) over the scattered frequency gives

$$
\int \mathrm{d} \omega_{2} f\left(\omega_{2}, \omega_{1} \mid \tau\right)=4 \pi \operatorname{Re} \int_{0}^{\infty} \mathrm{d} \tau_{1} \mathrm{e}^{1 \omega_{1} \tau_{1}} \operatorname{Tr} d_{2} d_{2}^{\dagger} \mathrm{e}^{-1 L \tau}\left[\left(\mathrm{e}^{-\mathrm{i} L \tau_{1}}\left(d_{1} \rho\right)\right) d_{1}^{\dagger}\right]
$$

This equality is relevant to the case that the scattered radiation is recorded as a function of time, with no spectral resolution. It generalizes the theoretical result of Mukamel et al. [13].

Finally, the situation of irradiation with a broad-band spectrum is described by (3.8) integrated over $\omega_{1}$. The result is

$$
\int \mathrm{d} \omega_{1} f\left(\omega_{2}, \omega_{1} \mid \tau\right)=4 \pi \operatorname{Re} \int_{0}^{\tau} \mathrm{d} \tau_{3} \mathrm{e}^{-\mathrm{i} \omega_{2} \tau_{3}} \operatorname{Tr} d_{2} \mathrm{e}^{-1 L \tau_{3}}\left[d_{2}^{\dagger} \mathrm{e}^{-L\left(\tau-\tau_{3}\right)}\left(d_{1} \rho d_{1}^{\dagger}\right)\right] .
$$

In the next sections we simplify the general result in special cases.

\section{Time-dependent strengths of Rayleigh-Raman scattering and fluorescence}

In the remainder of this paper we ignore the effects due to the motion of the atom and to the possible degeneracy of the atomic states. Hence we consider a non-moving three-state atom. Furthermore we adopt a factorization approximation introduced and discussed in refs. 6 and 7, which has shown to be successful in describing the 
strengths of the Rayleigh or Raman scattering and of the collision-induced fluorescence. So we perform the average over the perturber bath in eq. (3.8) separately for the three time intervals occurring in each one of the three terms The result may be expressed in terms of correlation fucntions $C_{\mathrm{ab}}$, defined by

$$
\operatorname{Tr}_{\mathrm{p}} \rho_{\mathrm{p}} \mathrm{e}^{-1 L \tau} A_{\mathrm{ab}}=C_{\mathrm{ab}}(\tau) A_{\mathrm{ab}}
$$

where $\rho_{\mathrm{p}}$ is the density matrix of the perturber bath, $A_{\mathrm{ab}}$ is an operator between the states $|\mathrm{a}\rangle$ and $|\mathrm{b}\rangle$ of the scattering atom, and $\mathrm{Tr}_{\mathrm{p}}$ denotes the trace over the perturber states. The resulting simplified expression for the tıme-dependent redistribution function is

$$
\begin{aligned}
f\left(\omega_{2}, \omega_{1} \mid \tau\right)= & 2 p_{\mathrm{i}}\left|\mu_{\mathrm{ei}} \cdot \epsilon_{1}\right|^{2}\left|\mu_{\mathrm{ef}} \cdot \epsilon_{2}\right|^{2} \\
& \times\left[\operatorname{Re} \int_{0}^{\infty} \mathrm{d} \tau_{1} \int_{0}^{\infty} \mathrm{d} \tau_{2} \mathrm{e}^{\mathrm{i} \omega_{1} \tau_{1}+1\left(\omega_{1}-\omega_{2}\right) \tau_{2}-1 \omega_{2} \tau} C_{\mathrm{ef}}^{*}(\tau) C_{\mathrm{fi}}\left(\tau_{2}\right) C_{\mathrm{e} 1}\left(\tau_{1}\right)\right. \\
& +\operatorname{Re} \int_{0}^{\infty} \mathrm{d} \tau_{1} \int_{0}^{\tau} \mathrm{d} \tau_{3} \mathrm{e}^{1 \omega_{1} \tau_{1}-1 \omega_{2} \tau_{3}} C_{\mathrm{ef}}^{*}\left(\tau_{3}\right) \mathrm{e}^{-\gamma_{\mathrm{e}}\left(\tau-\tau_{3}\right)} C_{\mathrm{e} 1}\left(\tau_{1}\right) \\
& \left.+\operatorname{Re} \int_{0}^{\infty} \mathrm{d} \tau_{1} \int_{0}^{\tau} \mathrm{d} \tau_{3} \mathrm{e}^{-1 \omega_{1} \tau_{1}-1 \omega_{2} \tau_{3}} C_{\mathrm{ef}}^{*}\left(\tau_{3}\right) \mathrm{e}^{-\gamma_{\mathrm{e}}\left(\tau-\tau_{3}\right)} C_{\mathrm{el}}^{*}\left(\tau_{1}\right)\right],
\end{aligned}
$$

where $p_{1}$ is the fraction of atoms initially in the state $|1\rangle$, and $\gamma_{\mathrm{e}}$ is the decay rate of the excited state $|\mathrm{e}\rangle$ by inelastic collisions and by spontaneous emission. The Fourier-Laplace transform of the correlation function $C_{\mathrm{ab}}(\tau)$ determines a normalized profile $P_{\mathrm{ab}}(\omega)$ and a corresponding dispersion function, according to the relation

$$
P_{\mathrm{ab}}(\omega)-1 Q_{\mathrm{ab}}(\omega)=\frac{1}{\pi} \int_{0}^{\infty} \mathrm{d} \tau \mathrm{e}^{1 \omega \tau} C_{\mathrm{ab}}(\tau)
$$

After substituting (4.3) into (4.2) we obtain

$$
\begin{aligned}
& f\left(\omega_{2}, \omega_{1} \mid \tau\right)=2 \pi^{2} p_{1}\left|\mu_{\mathrm{ei}} \cdot \epsilon_{1}\right|^{2}\left|\mu_{\mathrm{ef}} \cdot \epsilon_{2}\right|^{2} \\
& \times\left\{\operatorname{Re} \mathrm{e}^{-\mathrm{i} \omega_{2} \tau} C_{\mathrm{ef}}^{*}(\tau)\left[P_{\mathrm{fi}}\left(\omega_{1}-\omega_{2}\right)-1 Q_{\mathrm{fi}}\left(\omega_{1}-\omega_{2}\right)\right]\left[P_{\mathrm{e} 1}\left(\omega_{1}\right)-1 Q_{\mathrm{ei}}\left(\omega_{1}\right)\right]\right. \\
& \left.+\frac{2}{\pi} P_{\mathrm{ei}}\left(\omega_{1}\right) \operatorname{Re} \int_{0}^{\tau} \mathrm{d} \tau_{3} \mathrm{e}^{-\mathrm{i} \omega_{2} \tau_{3}} C_{\mathrm{ef}}^{*}\left(\tau_{3}\right) \mathrm{e}^{-\gamma_{\mathrm{e}}\left(\tau-\tau_{3}\right)}\right\} .
\end{aligned}
$$

The structure of the two terms in (4.4) is quite different. The time dependence of the first term is given by $\exp \left(-1 \omega_{2} \tau\right) C_{\mathrm{ef}}^{*}(\tau)$, which has an oscillatory behavior with the frequency $\omega_{2}-\omega_{\mathrm{ef}}$, and which decays at a rate of the order of the sum of the collisional and the natural line width of the transition from $|e\rangle$ to $|f\rangle$. As a function of $\omega_{2}$ this first term will turn out to be important for $\omega_{2} \simeq \omega_{1}-\omega_{\mathrm{fi}}$, where the profile $P_{\mathrm{fi}}$ is maximal, and at the resonant value $\omega_{2} \simeq \omega_{\text {ef }}$, where the oscillatory time behavior is absent. The integral over $\omega_{2}$ of the first term in (4.4) is zero for $\tau>0$, as one may show from (4.2). The second term in (4.4) contains as a function of $\omega_{2}$ a peak centered at $\omega_{2} \simeq \omega_{\mathrm{ef}}$, with a strength proportional to $P_{\mathrm{e} 1}\left(\omega_{1}\right) \exp \left(-\gamma_{\mathrm{e}} \tau\right)$. Hence the strength of this peak decays at the same rate as the population of the excited state $|\mathrm{e}\rangle$. 
If we substrtute (4.4) into (3.10), we obtain for the function $g_{2}$ determining the time-dependent fluorescence spectrum

$$
\begin{aligned}
g_{2}\left(\omega_{2}, t\right)= & K\left\{\pi^{2} \operatorname{Re} \int_{-\infty}^{\infty} \mathrm{d} \omega_{1}\left[P_{\mathrm{fi}}\left(\omega_{1}-\omega_{2}\right)-1 Q_{\mathrm{fi}}\left(\omega_{1}-\omega_{2}\right)\right]\left[P_{\mathrm{e} 1}\left(\omega_{1}\right)-1 Q_{\mathrm{el}}\left(\omega_{1}\right)\right] z\left(\omega_{2}, \omega_{1}, t\right)\right. \\
& \left.+2 \pi \int_{-\infty}^{\infty} \mathrm{d} \omega_{1} \int_{0}^{\infty} \mathrm{d} \tau P_{\mathrm{e} 1}\left(\omega_{1}\right) g_{1}\left(\omega_{1}, t-\tau\right) x\left(\omega_{2}, \tau\right)\right\},
\end{aligned}
$$

where we introduce for abbreviation

$$
\begin{aligned}
& K=n_{\mathrm{A}} \frac{\omega_{2}^{4}}{\hbar^{2} c^{4}} p_{1}\left|\mu_{\mathrm{e} 1} \cdot \epsilon_{1}\right|^{2}\left|\mu_{\mathrm{ef}} \cdot \epsilon_{2}\right|^{2}, \\
& z\left(\omega_{2}, \omega_{1}, t\right)=\frac{1}{\pi} \int_{0}^{\infty} \mathrm{d} \tau \mathrm{e}^{-1 \omega_{2} \tau} C_{\mathrm{ef}}^{*}(\tau) g_{1}\left(\omega_{1}, t-\tau\right), \\
& x\left(\omega_{2}, \tau\right)=\frac{1}{\pi} \operatorname{Re} \int_{0}^{\tau} \mathrm{d} \tau_{3} \mathrm{e}^{-1 \omega_{2}{ }^{2} 3} C_{\mathrm{ef}}^{*}\left(\tau_{3}\right) \mathrm{e}^{-\gamma_{\mathrm{e}}\left(\tau-\tau_{3}\right)} .
\end{aligned}
$$

The complex function $z\left(\omega_{2}, \omega_{1}, t\right)$ as a function of $\omega_{2}$ may be looked upon as a convolution of

$$
\frac{1}{\pi} \int_{0}^{\infty} \mathrm{d} \tau \mathrm{e}^{-1 \omega_{2} \tau} C_{\mathrm{ef}}^{*}(\tau)=P_{\mathrm{ef}}\left(\omega_{2}\right)+1 Q_{\mathrm{ef}}\left(\omega_{2}\right)
$$

and the real function

$$
y\left(\omega_{2}, \omega_{1}, t\right)=\frac{1}{\pi} \operatorname{Re} \int_{0}^{\infty} \mathrm{d} \tau \mathrm{e}^{-i \omega_{2} \tau} g_{1}\left(\omega_{1}, t-\tau\right) .
$$

Hence we write

$$
z\left(\omega_{2}, \omega_{1} ; t\right)=\int_{-\infty}^{\infty} \mathrm{d} \omega^{\prime}\left[P_{\mathrm{ef}}\left(\omega_{2}-\omega^{\prime}\right)+{ }_{1} Q_{\mathrm{ef}}\left(\omega_{2}-\omega^{\prime}\right)\right] y\left(\omega^{\prime}, \omega_{1}, t\right)
$$

The validity of (4.11) may be checked by substituting (4.9) and (4.10) in its right-hand side. The width of $y$ as a function of $\omega_{2}$ depends on the rate of change of the incident radiation. Its strength is given by

$$
\int \mathrm{d} \omega_{2} y\left(\omega_{2}, \omega_{1} ; t\right)=g_{1}\left(\omega_{1}, t\right)
$$

The real function $x\left(\omega_{2}, \tau\right)$ may likewise be written as a convolution of $P_{\mathrm{ef}}\left(\omega_{2}\right)$ and the function

$$
\frac{1}{\pi} \operatorname{Re} \int_{0}^{\tau} \mathrm{d} \tau_{3} \mathrm{e}^{-1 \omega \tau_{3}} \mathrm{e}^{-\gamma_{\mathrm{e}}\left(\tau-\tau_{3}\right)}=\frac{1}{\pi}\left(\gamma_{\mathrm{e}}^{2}+\omega^{2}\right)^{-1}\left(\gamma_{\mathrm{e}} \cos \omega \tau+\omega \sin \omega \tau-\gamma_{\mathrm{e}} \mathrm{e}^{-\gamma_{\mathrm{e}} \tau}\right)
$$


which for $\tau>0$ has a strength $\mathrm{e}^{-\gamma_{\mathrm{e}} \tau}$, and a w1dth of the order of the maximum of $\gamma_{\mathrm{e}}$ and $\tau^{-1}$. Hence the strength of $x$ is given by

$$
\int_{-\infty}^{\infty} \mathrm{d} \omega_{2} x\left(\omega_{2}, \tau\right)=\mathrm{e}^{-\gamma_{\mathrm{e}} \tau} \quad \text { for } \tau>0
$$

We consider the case of far-wing irradiation, and we shall derive expressions for the time-dependent strengths of the Rayleigh-Raman scattering and of the fluorescence. The frequency $\omega_{0}$ of the incident rariation has a fixed value in the far wing of the absorption line, so that

$$
\left|\omega_{0}-\omega_{\mathrm{ei}}\right| \gg \gamma_{\mathrm{ei}}
$$

We must expect that rapid intensity variations of the incident radiation effectively broaden its spectrum. Only when the rate of change of the incident intensity is small compared to $\left|\omega_{0}-\omega_{\mathrm{ei}}\right|$ can we expect that the two peaks in the scattered spectrum are well separated. Therefore we consider the situation that the rate of change of the incident intensity at time $t$ is slow compared to $\left|\omega_{0}-\omega_{\mathrm{e}_{1}}\right|$, and furthermore that this has been so since $t-t_{0}$, where $t_{0}$ is large compared to $\left|\omega_{0}-\omega_{\mathrm{el}_{1}}\right|^{-1}$.

If these conditions are met, the function $y$ defined by (4.10) has a width that is much smaller than $\left|\omega_{0}-\omega_{\mathrm{ei}}\right|$, both as a function of $\omega_{1}$ and $\omega_{2}$. Then the first term of (4.5) contributes to $g_{2}$ two well-separated peaks, one at $\omega_{2} \simeq \omega_{\mathrm{ef}}$, and one at $\omega_{2} \simeq \omega_{0}-\omega_{\mathrm{fi}}$. In order to evaluate the strengths of these two contributions, we use the asymptotic behavior of the dispersion functions [7]

$$
Q_{\mathrm{ab}}(\omega) \simeq \frac{-1 / \pi}{\omega-\omega_{\mathrm{ab}}}, \quad \text { if }\left|\omega-\omega_{\mathrm{ab}}\right| \gg \gamma_{\mathrm{ab}}
$$

In this asymptotic region $P_{\mathrm{ab}}$ is much smaller than $Q_{\mathrm{ab}}$. The asymptotic behavior of the function $z$ is found to be

$$
z\left(\omega_{2}, \omega_{1}, t\right)=\frac{-1 / \pi}{\omega_{0}-\omega_{\mathrm{ei}}} g_{1}\left(\omega_{1}, t\right) \text { if } \omega_{2} \simeq \omega_{0}-\omega_{\mathrm{fi}}
$$

for values of $\omega_{1}$ within the bandwidth of $g_{1}$. After applyıng similar considerations as in refs. 7 and 8 we find that the first term in (4.5) contributes to $g_{2}$ a peak at $\omega_{2} \simeq \omega_{0}-\omega_{\mathrm{f}_{1}}$ with a strength

$$
K \frac{1}{\left(\omega_{0}-\omega_{\mathrm{e} 1}\right)^{2}} \int \mathrm{d} \omega_{1} g_{1}\left(\omega_{1}, t\right)
$$

and a peak at $\omega_{2} \simeq \omega_{\text {ef }}$ with the negative strength

$$
-K \frac{1}{\left(\omega_{0}-\omega_{\mathrm{ei}}\right)^{2}} \int_{-\infty}^{\infty} \mathrm{d} \omega_{1} g_{1}\left(\omega_{1}, t\right)
$$

The total strength of the first term in (4.5) is zero, as it should. The second term in (4.5) contributes only to the fluorescence peak at $\omega_{2} \simeq \omega_{\mathrm{ef}}$. The strength of this contribution is

$$
2 \pi K \int_{0}^{\infty} \mathrm{d} \tau \mathrm{e}^{-\gamma} \mathrm{e}^{\tau} \int_{-\infty}^{\infty} \mathrm{d} \omega_{1} P_{\mathrm{e}}\left(\omega_{1}\right) g_{1}\left(\omega_{1}, t-\tau\right)
$$


As a conclusion of this section we find explicit expressions for the strength $S_{\mathrm{r}}$ of the Rayleigh-Raman peak and the strength $S_{\mathrm{f}}$ of the fluorescence. The resulting expressions

$$
S_{\mathrm{r}}(t)=K \frac{1}{\left(\omega_{0}-\omega_{\mathrm{el}}\right)^{2}} \int_{-\infty}^{\infty} \mathrm{d} \omega_{1} g_{1}\left(\omega_{1}, t\right)
$$

and

$$
S_{\mathrm{f}}(t)=K\left[2 \pi \int_{0}^{\infty} \mathrm{d} \tau \mathrm{e}^{-\gamma_{\mathrm{e}} \tau} \int_{-\infty}^{\infty} \mathrm{d} \omega_{1} P_{\mathrm{e} 1}\left(\omega_{1}\right) g_{1}\left(\omega_{1}, t-\tau\right)-\frac{1}{\left(\omega_{0}-\omega_{\mathrm{e} 1}\right)^{2}} \int_{-\infty}^{\infty} \mathrm{d} \omega_{1} g_{1}\left(\omega_{1}, t\right)\right]
$$

are valid under the condition that the incident radiation has a fixed frequency in the far wing of the absorption line, and that the rate of change of the incident intensity has been slow compared to the detuning during a tıme interval preceding $t$ that is large compared to the inverse detuning. In particular the expressions (4.18) and (4.19) are valid if at all times the rate of intensity change has been small compared to the detuning. This corresponds to what Courtens and Szoke [14] have termed the adiabatic situation. However, (4.18) and (4.19) are also applicable in the case of a very rapid rise and fall of an incident light pulse.

It is apparent from (4.18) that the strength of the Rayleigh or Raman scattering follows the light pulse, and is simply proportional to the instantaneous total incident intensity. On the other hand, (4.19) shows that the strength of the fluorescence depends on the incident spectrum during the recent past, over a time period $\gamma_{\mathrm{e}}^{-1}$.

The total time-dependent strength of the scattered light may be directly extracted from (4.5). We find

$$
\int \mathrm{d} \omega_{2} g_{2}\left(\omega_{2}, t\right)=2 \pi K \int_{0}^{\infty} \mathrm{d} \tau \mathrm{e}^{-\gamma_{\mathrm{e}} \tau} \int_{-\infty}^{\infty} \mathrm{d} \omega_{1} P_{\mathrm{e} 1}\left(\omega_{1}\right) g_{1}\left(\omega_{1}, t-\tau\right)
$$

This result is valid irrespective of the spectrum and the time behavior of the incident radiation.

\section{Explicit evaluation of time-dependent strengths}

In this section we derive explicit expressions for the strengths $S_{\mathrm{r}}(t)$ and $S_{\mathrm{f}}(t)$ of the two peaks in the fluorescence spectrum for an incident light pulse with an exponential rise and decay. We characterize the incident radiation by the positive-frequency part of the electric-field strength

$$
\begin{array}{ll}
E^{(+)}(t)=E_{0} \mathrm{e}^{-i \omega_{0} t} \mathrm{e}^{\beta t / 2}, & t<0, \\
E^{(+)}(t)=E_{0} \mathrm{e}^{-i \omega_{0} t}, & 0<t<T, \\
E^{(+)}(t)=E_{0} \mathrm{e}^{-i \omega_{0} t} \mathrm{e}^{-\beta(t-T) / 2}, & t>T .
\end{array}
$$

For illustrative purposes we wish to avoid complexities due to the overlap of the transient effects due to the rise and the fall of the pulse, so we assume that $T$ is large compared to $\gamma_{\mathrm{e}}^{-1}$ and to the inverse bandwidth of the absorption line. This ensures that the fluorescence spectrum has time to reach its stationary value during the pulse.

The total strength of the incident radiation is found from (2.7) and (5.1) 


$$
\int \mathrm{d} \omega_{1} g_{1}\left(\omega_{1}, t\right)= \begin{cases}I_{0} \mathrm{e}^{\beta t} & \text { for } t<0 \\ I_{0} & \text { for } 0<t<T \\ J_{0} \mathrm{e}^{-\beta(t-T)} & \text { for } t>T\end{cases}
$$

where

$$
I_{0}=\frac{c}{2 \pi}\left|E_{0}\right|^{2}
$$

is the peak intensity of the pulse. Evaluation of $S_{\mathrm{f}}(t)$ requires an expression for the overlap of the absorption profile $P_{\text {ei }}$ with a time integral involving $g_{1}$ at earlier times. If we calculate $g_{1}$ from (2.6), and substitute the result, the time integration can be done giving the lengthy result

$$
\begin{aligned}
& \int_{0}^{\infty} d \tau e^{-\gamma_{e} \tau} g_{1}\left(\omega_{1}, t-\tau\right) \\
& =I_{0} \frac{e^{\beta t}}{\beta+\gamma_{e}} \frac{\beta / 2 \pi}{(\beta / 2)^{2}+\left(\omega_{1}-\omega_{0}\right)^{2}}, \quad \text { if } t<0 \text {, } \\
& =I_{0} \frac{\beta / 2 \pi}{(\beta / 2)^{2}+\left(\omega_{1}-\omega_{0}\right)^{2}}\left\{\frac{\sin \left(\omega_{1} \cdots \omega_{0}\right) t}{\omega_{1}-\omega_{0}}+\frac{1}{\beta+\gamma_{\mathrm{e}}} \mathrm{e}^{-\gamma_{\mathrm{e}} t}\right. \\
& \left.+\frac{\beta / 2-\gamma_{e}}{\gamma_{e}^{2}+\left(\omega_{1}-\omega_{0}\right)^{2}}\left[e^{-\gamma_{e} t}-\cos \left(\omega_{1}-\omega_{0}\right) t+\gamma_{e} \frac{\sin \left(\omega_{1}-\omega_{0}\right) t}{\omega_{1}-\omega_{0}}\right]\right\}, \quad \text { if } 0<t<T \text {, } \\
& =I_{0}\left\{\frac{1}{\beta / 2-\gamma_{\mathrm{e}}}\left[\frac{\beta}{2 \gamma_{\mathrm{e}}} \mathrm{e}^{-\gamma_{\mathrm{e}}(t-T)}-\mathrm{e}^{-(\beta / 2)(t-T)}\right] \delta\left(\omega_{1}-\omega_{0}\right)\right. \\
& +\frac{\beta / 2 \pi}{(\beta / 2)^{2}+\left(\omega_{1}-\omega_{0}\right)^{2}}\left(\mathrm{e}^{-(\beta / 2)(t-T)} \frac{\sin \left(\omega_{1}-\omega_{0}\right)(t-T)}{\omega_{1}-\omega_{0}}-\frac{1}{\beta-\gamma_{\mathrm{e}}}\left(\mathrm{e}^{-\gamma_{\mathrm{e}}(t-T)}-\mathrm{e}^{-\beta(t-T)}\right)\right. \\
& +\frac{\gamma_{e}}{\left(\beta / 2-\gamma_{e}\right)^{2}+\left(\omega_{1}-\omega_{0}\right)^{2}}\left[e^{-(\beta / 2)(t-T)}\left\{\cos \left(\omega_{1}-\omega_{0}\right)(t-T)+\frac{\beta / 2-\gamma_{e}}{\omega_{1}-\omega_{0}} \sin \left(\omega_{1}-\omega_{0}\right)(t-T)\right\}\right. \\
& \left.\left.\left.-\mathrm{e}^{-\gamma_{\mathrm{e}}(t-r)}\right]\right\}\right), \quad \text { if } t>r
\end{aligned}
$$

In the calculation of this result we used that $T$ is sufficiently large to allow $g_{1}\left(\omega_{1}, t\right)$ to reach its stationary value $I_{0} \delta\left(\omega_{1}-\omega_{0}\right)$ long before $T$. The strengths of the expressions (5.4) may be evaluated from $(5.2)$

$$
\begin{aligned}
\int_{-\infty}^{\infty} \mathrm{d} \omega_{1} \int_{0}^{\infty} \mathrm{d} \tau \mathrm{e}^{-\gamma_{\mathrm{e}} \tau} g_{1}\left(\omega_{1}, t-\tau\right) & =I_{0} \frac{\mathrm{e}^{\beta t}}{\beta+\gamma_{\mathrm{e}}} & & \text { if } t<0, \\
& =\frac{I_{0}}{\gamma_{\mathrm{e}}}\left(1-\frac{\beta}{\beta+\gamma_{\mathrm{e}}} \mathrm{e}^{-\gamma_{e} t}\right) & & \text { If } 0<t<T,
\end{aligned}
$$




$$
=\frac{I_{0}}{\beta-\gamma_{\mathrm{e}}}\left[\frac{\beta}{\gamma_{\mathrm{e}}} \mathrm{e}^{-\gamma_{\mathrm{e}}(t-T)}-\mathrm{e}^{-\beta(t-T)}\right] \text { if } t>T .
$$

We wish to derive expressions for $S_{\mathrm{r}}$ and $S_{\mathrm{f}}$ in the two complementary limits that the rate of change $\beta$ is small or large

\subsection{Moderate time varnations}

First we consider the case that $\beta$ is small compared to the detuning

$$
\beta \ll\left|\omega_{0}-\omega_{\text {el }}\right|
$$

One notices from (4.15) that this does not preclude the possibility that $\beta$ be large compared to $\gamma_{\mathrm{e}}$. In this case the total strength (4.20) of the fluorescence is an overlap integral of the function (5.4), which is centered at $\omega_{0}$, and the profile $P_{\mathrm{el}}\left(\omega_{1}\right)$, which is centered at $\omega_{\mathrm{el}}$. Since the separation between the centers is large compared to the widths of these functions we may write to a good approximation [7]

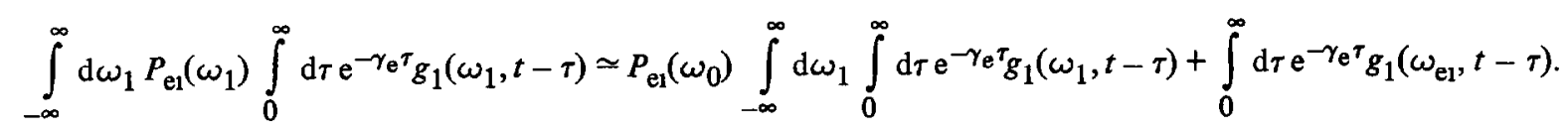

After substituting (5.7), (5.5) and (5.4) we obtain from (4.20) to second order in $\gamma_{\mathrm{e}} /\left(\omega_{0}-\omega_{\mathrm{e} 1}\right)$ and $\beta /\left(\omega_{0}-\omega_{\mathrm{e} 1}\right)$

$$
\begin{aligned}
& \int \mathrm{d} \omega_{2} g_{2}\left(\omega_{2}, t\right) \\
& =K I_{0} \frac{\mathrm{e}^{\beta t}}{\beta+\gamma_{\mathrm{e}}}\left[2 \pi P_{\mathrm{e} 1}\left(\omega_{0}\right)+\frac{\beta}{\left(\omega_{0}-\omega_{\mathrm{e} 1}\right)^{2}}\right] \quad \text { if } t<0, \\
& =K I_{0}\left[\left(1-\frac{\beta}{\beta+\gamma_{\mathrm{e}}} \mathrm{e}^{-\gamma_{\mathrm{e}} t}\right) \frac{2 \pi}{\gamma_{\mathrm{e}}} P_{\mathrm{e} 1}\left(\omega_{0}\right)+\frac{\beta}{\left(\beta+\gamma_{\mathrm{e}}\right)\left(\omega_{0}-\omega_{\mathrm{e} 1}\right)^{2}} \mathrm{e}^{-\gamma_{\mathrm{e}} t}\right] \\
& =\frac{K I_{0}}{\beta-\gamma_{\mathrm{e}}}\left[\left(\frac{\beta}{\gamma_{\mathrm{e}}} \mathrm{e}^{-\gamma_{\mathrm{e}}(t-T)}-\mathrm{e}^{-\beta(t-T)}\right) 2 \pi P_{\mathrm{e} 1}\left(\omega_{0}\right)-\left(\mathrm{e}^{-\gamma_{\mathrm{e}}(t-T)}-\mathrm{e}^{-\beta(t-T)} \frac{\beta}{\left(\omega_{0}-\omega_{\mathrm{e} 1}\right)^{2}}\right]\right. \\
& \text { if } 0<t<T,
\end{aligned}
$$

The strengths $S_{\mathrm{r}}$ and $S_{\mathrm{f}}$ of the peaks in the fluorescence spectrum are now directly found from (4.18), (4.19), (5.2) and (5.8). For abbrevation we introduce the notation

$$
R=K I_{0} \frac{1}{\left(\omega_{0}-\omega_{\mathrm{e} 1}\right)^{2}}
$$




$$
F=K I_{0}\left[\frac{2 \pi}{\gamma_{\mathrm{e}}} P_{\mathrm{e} 1}\left(\omega_{0}\right)-\frac{1}{\left(\omega_{0}-\omega_{\mathrm{e} 1}\right)^{2}}\right]
$$

for the stationary values of $S_{\mathrm{r}}$ and $S_{\mathrm{f}}$. Then the time-dependent strengths are

$$
\begin{aligned}
& S_{\mathrm{r}}(t)= \begin{cases}\mathrm{e}^{\beta t} R & \text { if } t<0 \\
R & \text { if } 0<t<T, \\
\mathrm{e}^{-\beta(t-T)} R & \text { if } t>T\end{cases} \\
& S_{\mathrm{f}}(t)= \begin{cases}\frac{\gamma_{\mathrm{e}}}{\beta+\gamma_{\mathrm{e}}} \mathrm{e}^{\beta t} F & \text { if } t<0 \\
{\left[1-\frac{\beta}{\beta+\gamma_{\mathrm{e}}} \mathrm{e}^{-\gamma_{\mathrm{e}} t}\right] F} & \text { if } 0<t<T . \\
\frac{1}{\beta-\gamma_{\mathrm{e}}}\left[\beta \mathrm{e}^{-\gamma_{\mathrm{e}}(t-T)}-\gamma_{\mathrm{e}} \mathrm{e}^{-\beta(t-T)}\right] F & \text { if } t>T\end{cases}
\end{aligned}
$$

It is interesting to note that $S_{\mathrm{f}}(t)$ as given by $(5.12)$ may be expressed in the general form

$$
S_{\mathrm{f}}(t)=K \gamma_{\mathrm{e}} \int_{-\infty}^{\infty} \mathrm{d} \omega_{1} \int_{0}^{\infty} \mathrm{d} \tau \mathrm{e}^{-\gamma_{\mathrm{e}} \tau} g_{1}\left(\omega_{1}, t-\tau\right)\left[\frac{2 \pi}{\gamma_{\mathrm{e}}} P_{\mathrm{ei}}\left(\omega_{0}\right)-\frac{1}{\left(\omega_{0}-\omega_{\mathrm{ei}}\right)^{2}}\right]
$$

as is obvious from a comparison of (5.5) and (5.12). This result suggests that (5.13) is generally valid if the rate of change of the incident intensity is small compared to the detuning. In fact, one may show this to be true after taking the proper limits in eq. (4.19).

As a result of the inequality (5.6) it is possible to choose the filter bandpass width $\Gamma$ to be small compared to the detuning, so that the two peaks may be separately observed, while at the same time $\Gamma$ is large compared to $\beta$, so that the time resolution suffices to observe the time behavior found in (5.11) and (5.12).

Fig. 1 shows the time behavior of the strengths $S_{\mathrm{r}}(t)$ and $S_{\mathrm{f}}(t)$ for different values of $\beta$. The strength $S_{\mathrm{r}}$ of the Rayleigh-Raman peak is proportional to the instantaneous pulse intensity at all times. If $\beta \ll \gamma_{e}$, the same is true for the strength $S_{\mathrm{f}}$ of the fluorescence. This corresponds to the quasistationary case. If $\beta \gtrsim \gamma_{\mathrm{e}}$, the strength $S_{\mathrm{f}}(t)$ does not follow the light pulse adiabatically, but it lags behind by a delay time $\gamma_{e}^{-1}$, due to the finite lifetime of the excited state.

\subsection{Rapid time variations}

We now turn to the case that $\beta$ is not much smaller than the detuning $\left|\omega_{0}-\omega_{\mathrm{ei}}\right|$. As a result of (4.15) we can then be sure that $\beta$ is much larger than $\gamma_{\mathrm{ei}}$ and $\gamma_{\mathrm{e}}$. In order to observe the two peaks separately, one has to use a spectrometer with a passband width $\Gamma$ that is much smaller than the detuning, and hence also smaller than $\beta$. The resolution time $\Gamma^{-1}$ is then too large to observe contributions in the strength that vary at the rate $\beta$. From (2.19) one notices that the observed strengths $\bar{S}_{\mathrm{r}}$ and $\bar{S}_{\mathrm{f}}$ of the peaks are related to the calculated strengths $S_{\mathrm{r}}$ and $S_{\mathrm{f}}$ 


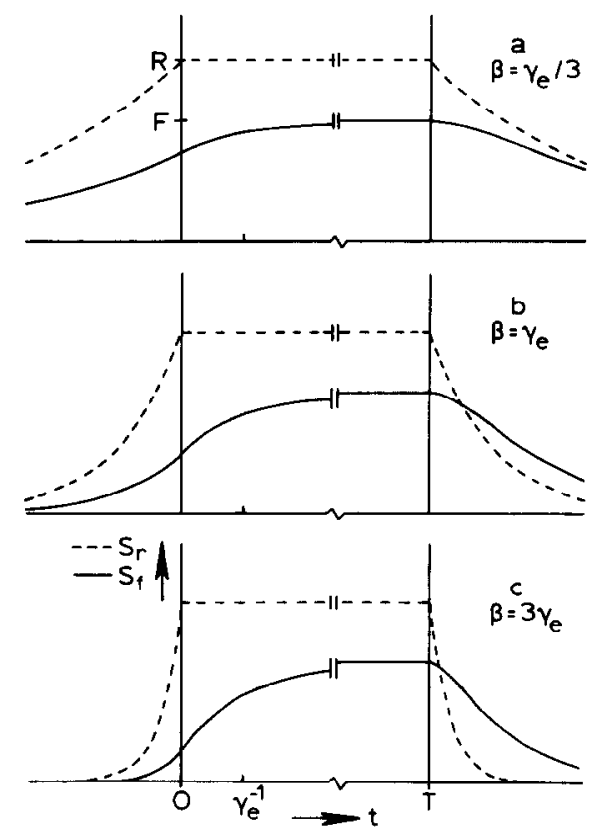

Fig 1. Time behavior of the strength $S_{\mathrm{r}}$ of the Rayleigh or Raman scattering and the strength $S_{\mathrm{f}}$ of the fluorescence for different values of the rise and decay rate $\beta$ of the incident intensity. These curves are valid when $\beta$ is much smaller than the detuning of the incident frequency from resonance The broken curve represents $S_{\mathrm{r}}$, the solid curve is $S_{\mathrm{f}}$ The values of $\beta$ are (a) $\beta=\gamma_{\mathrm{e}} / 3$, (b) $\beta=\gamma_{\mathrm{e}}$, (c) $\beta=3 \gamma_{\mathrm{e}}$. The Rayleigh-Raman strength $S_{\mathrm{r}}$ is proportional to the incident intensity at all times, the fluorescence strength $S_{\mathrm{f}}$ lags behind by a time $\gamma_{\mathrm{e}}^{-1}$ The curves in this figure correspond to eqs. (5.11) and (5.12).

according to

$$
\begin{aligned}
& \bar{S}_{\mathrm{r}}(t)=\int_{0}^{\infty} \mathrm{d} \tau \Gamma \mathrm{e}^{-\Gamma \tau} S_{\mathrm{r}}(t-\tau), \\
& \bar{S}_{\mathrm{f}}(t)=\int_{0}^{\infty} \mathrm{d} \tau \Gamma \mathrm{e}^{-\Gamma \tau} S_{\mathrm{f}}(t-\tau) .
\end{aligned}
$$

This indicates that variations of $S_{\mathrm{r}}$ and $S_{\mathrm{f}}$ that are more rapıd than $\Gamma$ are washed out by this time-smoothing integral.

Eqs. (4.18) and (4.19) for $S_{\mathrm{r}}$ and $S_{\mathrm{f}}$ are valid outside transient regions of the order of $\left|\omega_{0}-\omega_{\mathrm{el}}\right|^{-1}$ around $t=0$ and $t=T$, where the peaks may be 1 ll separated. However, these regions contribute negligibly to $\bar{S}_{\mathrm{r}}$ and $\bar{S}_{\mathrm{f}}$, which allows us to apply (4.18) and (4.19) together with (5.14) and (5.15) in order to evaluate the observed strengths. For the same reason the rapidly varying terms in (5.2) and (5.4) are negligible. Hence we ignore (5.2) for $t<0$ and for $t>T$.

The expressions (5.4) deserve a little more attention. The expression for $t<0$ can again be simply 1gnored. The expression for $0<t<T$ separates after a transient time $\left|\omega_{1}-\omega_{\mathrm{ei}}\right|^{-1}$ in a broad-band term

$$
I_{0} \frac{\beta / 2 \pi}{(\beta / 2)^{2}+\left(\omega_{1}-\omega_{0}\right)^{2}} \frac{1}{\beta+\gamma_{\mathrm{e}}} \mathrm{e}^{-\gamma_{\mathrm{e}} t}
$$


with a strength $I_{0} \exp \left(-\gamma_{\mathrm{e}} t\right) /\left(\beta+\gamma_{\mathrm{e}}\right)$, and several narrow-band terms. From (5.5) we conclude that the narrowband terms have the complementary strength $I_{0}\left(1-\exp \left(-\gamma_{\mathrm{e}} t\right)\right) / \gamma_{\mathrm{e}}$. Likewise the expression (5.4) for $t>T$ separates in a broad-band term and a narrow-band term. In the limit $\beta \gg \gamma_{e}$ the broad-band term is approximated by

$$
\frac{-I_{0} / 2 \pi}{(\beta / 2)^{2}+\left(\omega_{1}-\omega_{0}\right)^{2}} \mathrm{e}^{-\gamma_{\mathrm{e}}(t-T)}
$$

with the negative strength $-I_{0} \exp \left(-\gamma_{\mathrm{e}}(t-T)\right) / \beta$. From (5.5) one checks that after a transient time $\beta^{-1}$ the strength of the narrow band in the limit $\beta \gg \gamma_{\mathrm{e}}$ is $I_{0} \exp \left(-\gamma_{\mathrm{e}}(t-T)\right) / \gamma_{\mathrm{e}}$. The overlap of $P_{\mathrm{el}}$ with the narrowband terms is found in the same manner as applied in (5.7), while the overlap with the broad-band contribution is approximated by using the equation

$$
\int \mathrm{d} \omega_{1} P_{\mathrm{e} 1}\left(\omega_{1}\right) \frac{\beta / 2 \pi}{(\beta / 2)^{2}+\left(\omega_{1}-\omega_{0}\right)^{2}} \simeq \frac{\beta / 2 \pi}{(\beta / 2)^{2}+\left(\omega_{0}-\omega_{\mathrm{e} 1}\right)^{2}} .
$$

Ignoring the transient tıme domains, we obtain in this approximation for the total strength of the scattered light

$$
\begin{aligned}
& \int \mathrm{d} \omega_{2} g_{2}\left(\omega_{2}, t\right) \\
& \simeq 0 \quad \text { if } t<0 \text {, } \\
& \simeq K I_{0}\left[\left(1-\mathrm{e}^{-\gamma_{\mathrm{e}} t}\right) \frac{2 \pi}{\gamma_{\mathrm{e}}} P_{\mathrm{el}}\left(\omega_{0}\right)+\mathrm{e}^{-\gamma_{\mathrm{e}} t} \frac{1}{(\beta / 2)^{2}+\left(\omega_{0}-\omega_{\mathrm{e} 1}\right)^{2}}\left(1+\frac{\beta^{2} / 2}{\left(\omega_{0}-\omega_{\mathrm{el}}\right)^{2}}\right)\right] \\
& \text { if } 0<t<T \text {, } \\
& \simeq K I_{0} \mathrm{e}^{-\gamma_{\mathrm{e}}(t-T)}\left[\frac{2 \pi}{\gamma_{\mathrm{e}}} P_{\mathrm{el}}\left(\omega_{0}\right)-\frac{1}{(\beta / 2)^{2}+\left(\omega_{0}-\omega_{\mathrm{e} 1}\right)^{2}}\right] \\
& \text { if } t>T \text {. }
\end{aligned}
$$

In the same approximation $S_{\mathrm{I}}$ and $S_{\mathrm{f}}$ are found to be

$$
S_{\mathrm{r}}(t) \simeq \begin{cases}0 & \text { if } t<0 \\ R & \text { if } 0<t<T \\ 0 & \text { if } t>T\end{cases}
$$

and

$$
S_{\mathrm{f}}(t) \simeq \begin{cases}0 & \text { if } t<0 \\ \left(1-\mathrm{e}^{-\gamma_{\mathrm{e}} t}\right) F+\mathrm{e}^{-\gamma_{\mathrm{e}} t} \lambda R & \text { if } 0<t<T, \\ \mathrm{e}^{-\gamma_{\mathrm{e}}(t-T)}[F+\lambda R] & \text { if } t>T\end{cases}
$$


where $R$ and $F$ are given by (5.9) and (5.10), and where $\lambda$ is

$$
\lambda=\frac{(\beta / 2)^{2}}{(\beta / 2)^{2}+\left(\omega_{0}-\omega_{\mathrm{el}}\right)^{2}} .
$$

The parameter $\lambda$ is negligible if $\beta$ is much smaller than the detuning, and it approaches unity in the opposite limit that $\beta$ greatly exceeds $\left|\omega_{0}-\omega_{\mathrm{el}}\right|$ One notices in (5.18) and (5.19) that the neglect of the transient time domains gives rise to a discontinuous step $\pm R$ in $S_{\mathrm{r}}$, and a step $\lambda R$ in $S_{\mathrm{f}}$ at $t=0$ and $t=T$. This shows that these changes occur in fact at the rapid time scale measured by $\left|\omega_{0}-\omega_{\mathrm{el}}\right|$ and by $\beta$.

The physically observed strength $\overline{S_{\mathrm{r}}}$ and $\bar{S}_{\mathrm{f}}$ are found after substituting (5.18) and (5.19) in (5.14) and (5.15). We choose the spectral resolution $\Gamma$ to be large compared to the absorption linewidth and $\gamma_{\mathrm{e}}$, so that $a$ fortzori $\Gamma$ is large compared to $T^{-1}$. The result is

$$
\bar{S}_{\mathrm{r}}(t) \simeq \begin{cases}0 & \text { if } t<0 \\ \left(1-\mathrm{e}^{-\Gamma t}\right) R & \text { if } 0<t<T, \\ \mathrm{e}^{-\Gamma(t-T)} R & \text { if } t>T\end{cases}
$$

and

$$
\bar{S}_{\mathrm{f}}(t)\left\{\begin{array}{cc}
\simeq 0 & \text { if } t<0, \\
\simeq\left[1-\frac{1}{\Gamma-\gamma_{\mathrm{e}}}\left(\Gamma \mathrm{e}^{-\gamma_{\mathrm{e}} t}-\gamma_{\mathrm{e}} \mathrm{e}^{-\Gamma t}\right)\right] F+\frac{\Gamma}{\Gamma-\gamma_{\mathrm{e}}}\left(\mathrm{e}^{-\gamma_{\mathrm{e}} t}-\mathrm{e}^{-\Gamma t}\right) \lambda R \\
\quad \text { If } 0<t<T, \\
\simeq \frac{1}{\Gamma-\gamma_{\mathrm{e}}}\left[\Gamma \mathrm{e}^{-\gamma_{\mathrm{e}}(t-T)}-\gamma_{\mathrm{e}} \mathrm{e}^{-\Gamma(t-T)}\right] F+\frac{\Gamma}{\Gamma-\gamma_{\mathrm{e}}}\left[\mathrm{e}^{-\gamma_{\mathrm{e}}(t-T)}-\mathrm{e}^{-\Gamma(t-T)}\right] \lambda R \\
\quad \text { if } t>T .
\end{array}\right.
$$

The observed strength of the Rayleigh-Raman peak shows a nse and decay determined by the resolution time $\Gamma^{-1}$. The fluorescence peak at $\omega_{2} \simeq \omega_{\text {ef }}$ shows variations on two time scales. In the usual case that $\Gamma$ is much larger than $\gamma_{\mathrm{e}}, \overline{S_{\mathrm{f}}}$ shows at $t=0$ a fast rise with the amount $\lambda R$, and subsequently slowly approaches its stationary value $F$. At $t=T, \bar{S}_{\mathrm{f}}$ again shows a fast rise of about $\lambda R$, and then decays to zero on the slow time scale. The amount of these fast rises increases with the pulse decay rate $\beta$. This may be understood as a result of the effective broadening of the incident spectrum by rapid intensity varıations. It is remarkable that these rises in $\bar{S}_{\mathrm{f}}$ occur on the rapid time scale, and that their rates are not restricted by the lifetime of the excited state.

In fig. 2 we show $\bar{S}_{\mathrm{f}}$ in the case that $\Gamma=10 \gamma_{\mathrm{e}}$ for vanous values of $\lambda R / F$. This parameter decreases with increasing perturber pressure and it increases with $\beta$. It is apparent from fig. 2 that $\bar{S}_{\mathrm{f}}$ attains its maximum value after the pulse is over. The sharp rise of $\bar{S}_{\mathrm{f}}$ after $t=T$ is more than counterbalanced by the rapid decay of the RayleighRaman peak strength. One notices from eqs. (5.21) and (5.22) that the observed total strength $\overline{S_{\mathrm{r}}}+\bar{S}_{\mathrm{f}}$ of the scattered light shows a fast decrease by about $(1-\lambda) R$ at $t=T$. The slowly decaying component after $t=T$ is entirely due to $\overline{S_{\mathrm{f}}}$, and its strength is $F+\lambda R$. In the limit $\beta \rightarrow \infty$ or $\lambda \rightarrow 1$, corresponding to a square pulse, it is the total stationary intensity $F+R$ of the scattered light that decays at the slow rate $\gamma_{\mathrm{e}}$, but the frequency of the scattered light after $t=T$ is centered at the resonance frequency $\omega_{2} \simeq \omega_{\mathrm{ef}}$. 


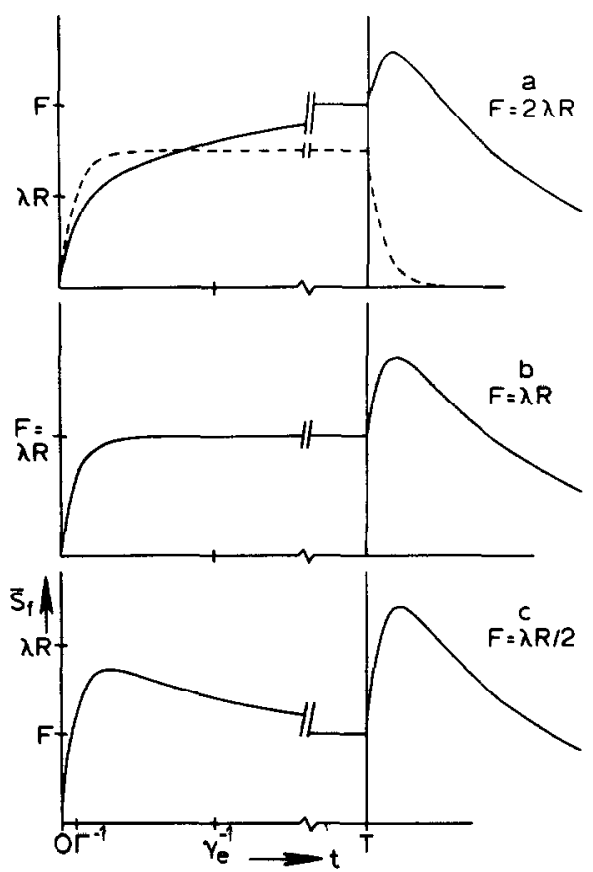

Fig. 2. Time behavior of the observed strength $\bar{S}_{\mathrm{f}}$ of the fluorescence for different values of $\lambda R / F$. These curves are valld when $\beta$ is of the same order of magnitude as the detuning of the incident frequency from resonance or larger. The broken curve in (a) shows the observed Rayleigh-Raman strength in the case that $\lambda=2 / 3$. The solid curves represent $\bar{S}_{f}$, for $(a) \lambda R / F=\frac{1}{2},(b) \lambda R / F=1$, (c) $\lambda R / F=2$. The passband width of the spectrometer $1 \mathrm{~s} \Gamma=10 \gamma_{\mathrm{e}}$ Immediately after $t=0 \overrightarrow{S_{\mathrm{f}}}$ increases with the rate $\Gamma$ by an amount of the order of $\lambda R$, and subsequently it approaches its stationary value $F$-at the rate $\gamma_{\mathrm{e}}$. After $t=T \bar{S}_{\mathrm{f}}$ agann mereases by an amount of about $\lambda R$, then it decays to zero at the slow rate $\gamma_{e}$. The curves in this figure represent eqs (5.21) and (5.22).

\section{Conclusions}

We denve a general expression for the time-dependent spectrum of light scattered from atoms in a perturber gas. The result is expressed in eqs. (3.7)-(3.10) in terms of a time-dependent redistribution function $f\left(\omega_{2}, \omega_{1} \mid \tau\right)$. From this general result we evaluated the time-dependent strengths $S_{\mathrm{r}}(t)$ and $S_{\mathrm{f}}(t)$ of Rayleigh-Raman scattering and of fluorescence in the case that the detuning of the incident frequency from resonance is large. The resulting expressions (4.18) and (4.19) are valid for times at which the rate of change of the incident intensity has been small compared to the detuning over a period of time that is larger than the inverse detuning. These expressions have yet to be time-smoothed over the resolution time of the spectrometer.

When the urradiated pulse has an exponential rise and decay, the strengths $S_{\mathrm{r}}$ and $S_{\mathrm{f}}$ are given by eqs. (5.11) and (5.12) if the rise and decay times are large compared to the inverse detuning. These results are shown in fig. 1. In the complementary case that the rise and decay rates are not small compared to the detuning, the observed strengths are given in eqs. (5.21) and (5.22), and plotted in fig. 2.

We did not incorporate saturation effects. The potartzatton dependence of the scattered intensity 15 inctuded $\mathrm{m}$ the general results in section 3, but it is ignored in sections 4 and 5 . Our results should describe a spectrally resolved version of the experiments of Rousseau et al. [10-12]. 


\section{References}

[1] J. L Carlsten and A. Szoke, Phys. Rev. Lett. 36 (1976) 667.

[2] J. L. Carlsten and A. Szoke, J. Phys. B. Atom. Mol. Phys. 9 (1976) L231.

[3] J. L. Carlsten, A Szoke and M. G. Raymer, Phys. Rev A15 (1977) 1029.

[4] D. L. Huber, Phys Rev. 178 (1969) 93

[5] A. Omont, E. W. Smith and J. Cooper, Astrophys J. 175 (1972) 185.

[6] G. Nienhuis and F. Schuller, Physica 92C (1977) 397

[7] G. Nienhuis and F. Schuller, Physica 92C (1977) 409

[8] G. Nienhu1s, Physica 93C (1978) 393.

[9] G. Nienhu1s, Physica 95C (1978) 266.

[10] P. F. Williams, D. L Rousseau and S H. Dworetsky, Phys. Rev. Lett. 32 (1974) 196.

[11] D. L. Rousseau, G D Patterson and P. F. Willams, Phys. Rev. Lett. 34 (1975) 1306

[12] D L Rousseau and P F. Willams, J. Chem. Phys. 64 (1976) 3519

[13] S. Mukamel, A Ben-Reuven and J Jortner, Phys. Rev. A12 (1975) 947

[14] E. Courtens and A. Szoke, Phys. Rev A15 (1977) 1588

[15] J. H. Eberly and K. Wódkıewicz, J. Opt. Soc. Am. 67 (1977) 1252.

[16] B. Renaud, R. M. Whitley and C. R. Stroud Jr., J. Phys. B. Atom Mol. Phys. 10 (1977) 19.

[17] M. Born and E. Wolf, Prunciples of Optics (Pergamon Press, Oxford, 1975). 